\title{
Binary pulsar B1259-63 spectrum evolution: detailed study
}

\author{
Marta Dembska, Jarosław Kijak and Wojciech Lewadowski \\ Kepler Institute of Astronomy, University of Zielona Góra \\ Lubuska 2, 65-265 Zielona Góra, Poland \\ email: marta@astro.ia.uz.zgora.pl
}

\begin{abstract}
We studied the radio spectrum of PSR B1259-63 in an unique binary with Be star LS 2883 and showed that the shape of the spectrum depends on the orbital phase. We proposed a qualitative model which explains this evolution. We considered two mechanisms that might influence the observed radio emission: free-free absorption and cyclotron resonance. Recently published results have revealed a new aspect in pulsar radio spectra. There were found objects with turnover at high frequencies in spectra, called gigahertz-peaked spectra (GPS) pulsars. Most of them adjoin such interesting environments as HII regions or compact pulsar wind nebulae $(\mathrm{PWN})$. Thus, it is suggested that the turnover phenomenon is associated with the environment than being related intrinsically to the radio emission mechanism. Having noticed the apparent resemblance between the B1259-63 spectrum and the GPS, we suggest that the same mechanisms should be responsible for both cases. Therefore, the case of B1259-63 can be treated as a key factor to explain the GPS phenomenon observed for the solitary pulsars with interesting environments and also another types of spectra (e.g. with break).
\end{abstract}

Keywords. pulsars: general, individual (B1259-63) - stars: winds, outflows - ISM: general, magnetic fields - radiations mechanism: non-thermal

\section{The gigahertz-peaked spectra pulsars}

Generally, the observed radio spectra of most pulsars can be modelled as a power law with negative spectral indices of about -1.8 (Maron et al. 2000). If a pulsar can be observed at frequencies low enough (i.e. 100-600 MHz), it may also show a low-frequency turnover in its spectrum (Sieber 1973; Malofeev et al. 1994). On the other hand, Lorimer et al. (1995) mentioned three pulsars which have positive spectral indices in the frequency range 300-1600 MHz. Later, Maron et al. (2000) re-examined spectra of these pulsars taking into account the data obtained at higher frequencies (above $1.6 \mathrm{GHz}$ ) and consequently were the first to demonstrate a possible existence of spectra with turnover at high frequencies, about $1 \mathrm{GHz}$. Kijak et al. (2011a) provided a definite evidence for a new type of pulsar radio spectra. These spectra show the maximum flux above $1 \mathrm{GHz}$, while at higher frequencies the spectra look like a typical pulsar spectrum. At lower frequencies (below $1 \mathrm{GHz}$ ), the observed flux decreases, showing a positive spectral index (Kijak et al. 2011a). They called these objects the gigahertz-peaked spectra (GPS) pulsars. A frequency at which such a spectrum shows the maximum flux was called the peak frequency. Kijak et al. (2011a) also indicated that the GPS pulsars are relatively young objects, and they usually adjoin such interesting environments as HII regions or compact pulsar wind nebulae. Additionally, some of them seem to be coincident with the known but sometimes unidentified X-ray sources from third EGRET Catalogue or HESS observations. We can assume that the GPS appearance owes to the environmental conditions around the neutron stars rather than to the radio emission mechanism. 
B1259-63, J1302-6350

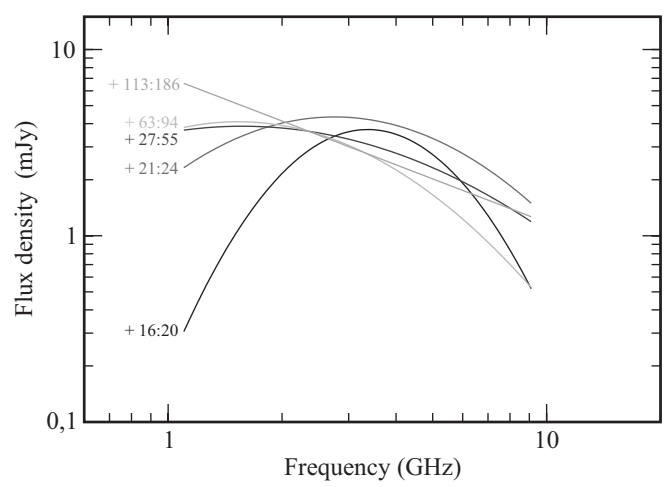

B1259-63, J1302-6350

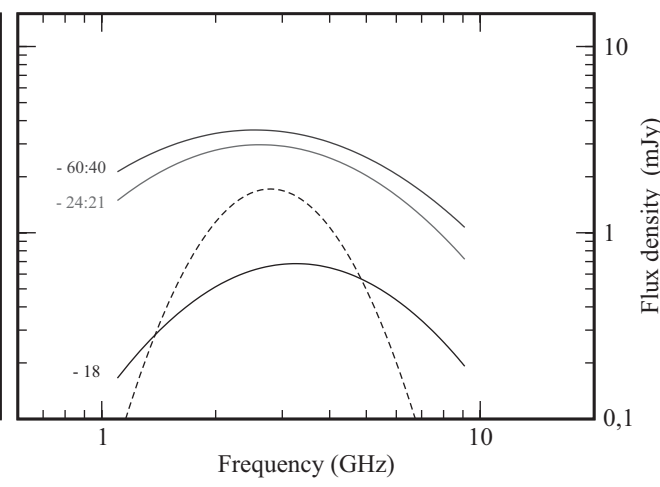

Figure 1. The fits to the B1259-63 spectra for the each orbital phase range (Kijak et al. 2011b), from $60 \mathrm{~d}$ prior to periastron (left panel) up to $186 \mathrm{~d}$ after it (right panel).

\section{PSR B1259-63 spectrum evolution}

PSR B1259-63 was also listed by Lorimer et al. (1995) as a pulsar with positive spectral index. Therefore, it seems a natural candidate to be classified as the GPS pulsar. This pulsar is in an unique binary with a massive main-sequence Be star. PSR B1259-63 has a short period of $48 \mathrm{~ms}$ and a characteristic age of $330 \mathrm{kyr}$. Its average dispersion measure (DM) is about $147 \mathrm{pc} \mathrm{cm}^{-3}$ and the corresponding distance is about $2.75 \mathrm{kpc}$. The companion star LS 2883 is a 10-mag massive Be star with a mass of about $10 \mathrm{M}_{\odot}$ and a radius of $6 \mathrm{R}_{\odot}$. Be stars are generally believed to have a hot tenuous polar wind and a cooler high-density equatorial disc. The PSR B1259-63/LS 2883 emits unpulsed non-thermal emission over a wide range of frequencies ranging between radio and $\gamma$-rays, and its flux varies with orbital phase. We studied the radio spectrum of B1259-63 (Kijak et al. 2011b). We analysed the available measurements of the pulsed flux obtained during three periastron passages (1997, 2000 and 2004). Our analysis showed that this pulsar undergoes a spectrum evolution due to orbital motion (see Fig. 1). We suggested that this effect is caused by the interaction of the radio waves with the Be star environment. While the eclipse itself can be naturally explained by free-free absorption in the stellar disc, the disc alone is not enough to explain the spectra evolution. In addition, we have shown that the peak frequency also depends on the orbital phase and therefore it varies with the changes of the pulsar environment. We argued that such behaviour can be explained by the radio-wave absorption in the magnetic field associated with the disc. We proposed a qualitative model which explains this evolution. We argued that the observed variation of the spectra is caused by a combination of two effects: the free-free absorption in the stellar wind and the cyclotron resonance in the magnetic field. This field is associated with the disc and is infused by the relativistic particles of the pulsar wind.

B1259-63 as key factor to explain the GPS phenomenon. Having noticed the apparent resemblance between the B1259-63 spectrum and the GPS, we suggested that the same mechanisms should be responsible for both cases (Kijak et al. 2011b). Thus, we can conclude that the GPS feature should be caused by some external factors rather than by the emission mechanism. On the other hand, the GPS pulsars are isolated radio pulsars and therefore, we cannot draw a direct analogy between the PSR B1259-63/LS 2883 system and the GPS pulsars, as the latter have no companion stars and/or discs. But the GPS pulsars apparently are surrounded by some kind of environment that can affect the spectra of those pulsars in the same way as the stellar wind affects the B1259-63 spectrum. All GPS pulsars have relatively high DMs that, in some 

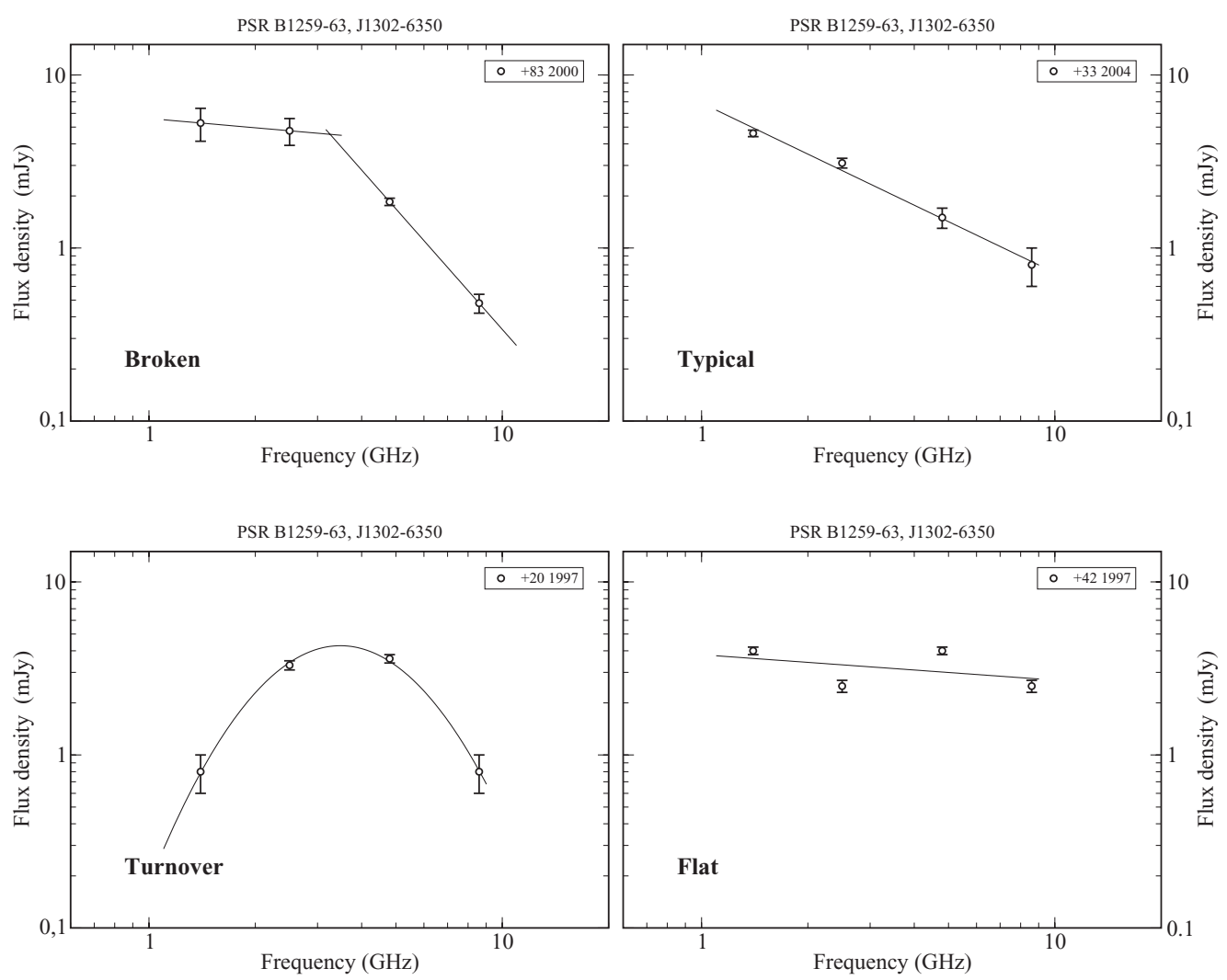

Figure 2. The fits to the B1259-63 spectra for chosen days. Each panel shows different type of spectra.

cases, are too large to be accounted for by the Galactic electron density, and thus, we can speculate that there is a quite high particle density in the vicinity of these pulsars (see also Kijak et al. 2011a). Thus, we believe that this binary system can hold the clue to the understanding of gigahertz-peaked spectra of isolated pulsars. The only difference could be an invariable shape of the GPS.

\section{PSR B1259-63 spectrum evolution: detailed study}

Using the same database (Johnston et al. 1999, Connors et al. 2002, Johnston et al. 2005) we constructed spectra for chosen observing days. We analysed the shapes of the B1259-63 spectrum at various orbital phase ranges as it was done previously (Kijak et al. 2011b) and obtained results are consistent with those for intervals. The flux at the given frequency apparently changes with orbital phases. When the pulsar is close to periastron, the flux generally decreases at all observed frequencies and the most drastic decrease is observed at the lowest frequency. Moreover, we noticed all types of radio pulsar spectra.

PSR B1259-63 is a object with relatively high dispersion measure which means that its transition frequency is very high. This implies that we definitely have to take into consideration both refractive (RISS) and diffractive (DISS) scintillations when analysing spectra for a given day. We used diffractive bandwidth $\Delta f_{\text {DISS }}$ and timescale $\Delta t_{\text {DISS }}$ from scintillation observations of the pulsar made far from periastron at $4.8 \mathrm{GHz}$ and 
8.4 GHz (McClure-Griffiths et al. 1998) to estimate values of these parameters at $1.4 \mathrm{GHz}$ and $2.4 \mathrm{GHz}$ assuming $\Delta t_{\text {DISS }} \propto f^{1.2} d^{-0.6}$, where $f$ and $d$ denote frequency and distance respectively. We estimated the values of $\Delta t_{\text {DISS }}$ to be ranging from $40 \mathrm{~s}$ at $1.4 \mathrm{GHz}$ to $360 \mathrm{~s}$ at $8.4 \mathrm{GHz}$ which suggests that diffractive scintillations should not affect the average flux measurements (observing sessions was usually 4 hours long). Roughly estimated refractive timescales vary from 12 hours at $8.4 \mathrm{GHz}$ to more than 20 days at $1.4 \mathrm{GHz}$. However, for lower frequencies the modulation index is relatively small which means lower uncertainty estimates when measuring flux. High frequency observations will be affected by refractive scintillations what leads to conclusion that flux values should be averaged over epochs and/or orbital phase intervals to be more reliable.

\section{Conclusions}

Close to the periastron point the spectra of B1259-63 resemble those of the GPS pulsars. The spectrum for the orbital epochs further from the periastron point are more consistent with typical pulsar spectra (i.e. power-law and broken). Moreover, detailed study of PSR B1259-63 spectra revealed the appearance of all types of spectral shapes, including a flat spectrum (see Fig. 2).

We believe that the case of B1259-63 can be treated as a key factor to our understanding of not only the GPS phenomenon (observed for the solitary pulsars with interesting environments) but also other types of untypical spectra as well (e.g. flat or broken spectra). This in turn would suggest, that the appearance of various non-standard spectra shapes in the general population of pulsars can be caused by peculiar environmental conditions.

\section{Acknowledgements}

MD is a scholar within Sub-measure 8.2.2 Regional Innovation Strategies, Measure 8.2 Transfer of knowledge, Priority VIII Regional human resources for the economy Human Capital Operational Programme co-financed by European Social Fund and state budget.

\section{References}

Connors, T. W., Johnston, S., Manchester, R. N., \& McConnell, D. 2002, MNRAS, 336, 1201

Johnston, S., Manchester, R. N., McConnell, D., \& Campbell-Wilson, D., 1999, MNRAS, 302, 277

Johnston, S., Ball, L., Wang, N., \& Manchester, R. N., 2005, MNRAS, 358, 1069

Kijak, J., Lewandowski, W., Maron, O., Gupta, Y., \& Jessner, A., 2011a, A\&A, 531, A16

Kijak, J., Dembska, M., Lewandowski, W., Melikidze, G., \& Sendyk, M., 2011b, MNRAS, 418, L114

Lorimer, D. R., Yates, J. A., Lyne, A. G., \& Gould, D. M., 1995, MNRAS, 273, 411

Malofeev, V. M., Gil, J. A., Jessner, A., et al. 1994, A\&A, 285, 201

Maron, O., Kijak, J., Kramer, M., \& Wielebinski, R., 2000, A\&\&A S, 147, 195

McClure-Griffiths, N. M., Johnston, S., Stinebring, D. R., \& Nicastro, L., ApJ, 1998, 492, L49

Sieber, W., 1973, A\&A, 28, 237 